\title{
Soluble RAGE attenuates Angll-induced endothelial hyperpermeability by disrupting HMGB1-mediated crosstalk between AT1R and RAGE
}

\author{
Jisu Jeong ${ }^{1,2}$, Jiye Lee ${ }^{2}$, Juyeon Lim²,2, Soyoung Cho ${ }^{1,2}$, Soyoung An², Myungeun Lee' ${ }^{2}$ Nara Yoon ${ }^{3}$, Miran Seo ${ }^{2}$,
} Soyeon Lim (1) ${ }^{4}$ and Sungha Park 2,5

\begin{abstract}
Increased endothelial permeability, one of the earliest signs of endothelial dysfunction, is associated with the development of cardiovascular diseases such as hypertension and atherosclerosis. Recent studies suggest that the receptor for advanced glycation end products (RAGE) regulates endothelial permeability in inflammation. In the present study, we investigated the regulatory mechanism of RAGE in endothelial hyperpermeability induced by angiotensin II (Ang II), a well-known inflammatory mediator, and the potential therapeutic effect of soluble RAGE (sRAGE), a decoy receptor for RAGE ligands. For in vitro studies, Ang II-treated human umbilical vein endothelial cells (HUVECs) were treated with siRNA specific to either RAGE or SRAGE to disrupt RAGE-mediated signaling. Endothelial permeability was estimated using FITC-labeled dextran 40 and a resistance meter. To evaluate intercellular junction disruption, VE-cadherin expression was examined by western blotting and immunocytochemistry. Ang II increased the expression of the Ang II type 1 receptor (AT1R) and RAGE, and this increase was inhibited by sRAGE. sRAGE prevented Ang II-induced VE-cadherin disruption in HUVECs. For in vivo studies, Ang II-infused, atherosclerosis-prone apolipoprotein E knockout mice were utilized. Endothelial permeability was assessed by Evans blue staining of the aorta. Ang II increased endothelial barrier permeability, and this effect was significantly attenuated by sRAGE. Our data demonstrate that blockade of RAGE signaling using sRAGE attenuates Ang II-induced endothelial barrier permeability in vitro and in vivo and indicate the therapeutic potential of SRAGE in controlling vascular permeability under pathological conditions.
\end{abstract}

\section{Introduction}

Endothelial cells play a critical role in maintaining the homeostasis of blood vessels. Regulation of the endothelial barrier is important for maintaining the integrity of cell-to-cell adhesion because increased endothelial permeability can lead to endothelial dysfunction, a common prelude to the development of vascular diseases such as atherosclerosis ${ }^{1}$. Endothelial hyperpermeability is also a

\footnotetext{
Correspondence: Soyeon Lim (slim724@cku.ac.kr) or Sungha Park (shpark0530@yuhs.ac)

${ }^{1}$ Graduate Program in Science for Aging, Yonsei University, Seoul 120-752, Korea

${ }^{2}$ Integrative Research Center for Cerebrovascular and Cardiovascular Diseases, Yonsei University College of Medicine, Seoul 120-752, Korea

Full list of author information is available at the end of the article.
}

significant problem in a number of conditions, such as vascular inflammation, ischemia-reperfusion injury, diabetes, and thrombosis and cancer ${ }^{2}$. Excessive inflammatory stimuli, such as transforming growth factor-beta (TGF- $\beta$ ), thrombin, and tumor necrosis factor-alpha (TNF- $\alpha$ ), can trigger actin cytoskeleton reorganization via such events as filamentous actin (F-actin) disruption, stress fiber formation, and cytoskeletal contraction, leading to transendothelial flux via the widened intercellular space $^{2,3}$. Since reduced availability of nitric oxide due to high oxidative stress and inflammation can also cause endothelial hyperpermeability ${ }^{4,5}$, angiotensin II (Ang II) can be another key modulator of endothelial hyperpermeability, because the renin-angiotensin system (RAS)

\section{(c) The Author(s) 2019}

(c) Open Access This article is licensed under a Creative Commons Attribution 4.0 International License, which permits use, sharing, adaptation, distribution and reproduction cc in any medium or format, as long as you give appropriate credit to the original author(s) and the source, provide a link to the Creative Commons license, and indicate if changes were made. The images or other third party material in this article are included in the article's Creative Commons license, unless indicated otherwise in a credit line to the material. If material is not included in the article's Creative Commons license and your intended use is not permitted by statutory regulation or exceeds the permitted use, you will need to obtain permission directly from the copyright holder. To view a copy of this license, visit http://creativecommons.org/licenses/by/4.0/. 
can lead to both high oxidative stress and inflammation, causing abnormal NO metabolism ${ }^{6}$.

Ang II is an important vasoconstrictor in the RAS and exerts multiple functional effects on endothelial cells, including excessive oxidative stress, vascular inflammation, thrombosis, and remodeling of the actin cytoskeleton $^{7}$. Although Ang II is known to induce endothelial hyperpermeability ${ }^{8}$, and previous studies have shown that Rac and Rho kinase mediate Ang II-induced cytoskeletal remodeling ${ }^{9,10}$, the underlying mechanisms by which Ang II induces endothelial hyperpermeability have not been completely elucidated. The biological action of Ang II is mainly mediated by the Ang II type 1 receptor (AT1R), and recent studies have suggested a link between AT1R and the receptor for advanced glycation end products (RAGE) as an important signaling pathway in vascular smooth muscle cells and podocytes ${ }^{11,12}$.

RAGE is a multiligand signal transduction receptor that recognizes various endogenous ligands, such as high mobility group box 1 (HMGB1), advanced glycation end products (AGE), macrophage-1 antigen (Mac-1), and S100/calgranulins, and plays an important role in inducing inflammatory reactions through the activation of protein kinase C (PKC)/phosphoinositide 3-kinase (PI3K)/Akt, Janus kinase/signal transducer and activator of transcription (JAK/STAT), mitogen-activated protein kinase (MAPK), extracellular signal-regulated kinase (ERK), and Src/RhoA/Cdc42, leading to the activation of transcription factors such as NF- $\mathrm{kB}$ and Egr $1^{13}$. Regarding the role of RAGE signaling in endothelial dysfunction, studies showed that AGE increased endothelial permeability through the RAGE/Rho signaling pathway or Src phosphorylation in human umbilical vein endothelial cells (HUVECs) ${ }^{14,15}$ and that AGE-induced Src phosphorylation and barrier dysfunction were inhibited in the pulmonary microvascular endothelial cells of RAGE knockout mice ${ }^{14}$.

Although these findings provide circumstantial evidence of possible crosstalk between AT1R-mediated signaling and RAGE-mediated signaling in Ang II-induced endothelial hyperpermeability, this link has not been empirically examined, and the underlying mechanism remains elusive. Therefore, in the present study, we investigated the regulatory mechanism of RAGE in Ang II-induced endothelial hyperpermeability and the potential therapeutic effect of soluble RAGE (sRAGE), a decoy receptor for RAGE ligands.

\section{Materials and methods Chemicals and antibodies}

Purified human soluble RAGE-Fc fusion protein (sRAGE) was purchased from Y-biologics (Daejeon, Republic of Korea). Ang II, losartan, caffeic acid phenethyl ester (CAPE), and fluorescein isothiocyanate (FITC)- labeled dextran 40 were obtained from Sigma-Aldrich (St. Louis, MO, USA). Purified anti-HMGB1 antibody (651402) was purchased from BioLegend (San Diego, CA, USA). Anti-AT1R antibody (AAR-011) was purchased from Alomone Labs (Hadassah Ein Kerem, Israel). Anti-RAGE (ab3611), anti-mDia1 (ab129167), and anti-HMGB1 (ab18256) antibodies were purchased from Abcam (Cambridge, UK). Anti-Src (2109s), anti-phospho-Src (6943s, Tyr416), anti- $\beta$-catenin (8480s), anti-phospho $\beta$-catenin (5651s, Ser522), and anti-phospho-NF-kB (3033s, Ser536) antibodies were obtained from Cell Signaling Technology (Danvers, MA, USA). Anti-phospho-VE-cadherin (LSC357312, Tyr731) was purchased from LifeSpan Biosciences (Seattle, WA, USA). Anti-VE-cadherin (sc9989), anti-NF-kB (sc8008), anti- $\beta$-actin (sc47778), and antiGAPDH (sc32233) antibodies were purchased from Santa Cruz Biotechnology (Dallas, TX, USA).

\section{Cell culture}

HUVECs were purchased from Lonza and maintained in endothelial growth medium-2 (EGM-2, Lonza, Basel, Switzerland) supplemented with $2 \%(\mathrm{v} / \mathrm{v})$ fetal bovine serum (FBS) at $37^{\circ} \mathrm{C}$ in a humidified atmosphere containing $5 \% \mathrm{CO}_{2}$. HUVECs were cultured in $1.5 \%$ gelatincoated cell culture dishes, and cells at passages $4-8$ were used in the experiments.

\section{Permeability assay}

HUVECs were grown in the upper chambers of 24-well Transwell inserts $(0.4 \mu \mathrm{m}$ pore size; Corning, NY, USA) until a confluent monolayer was formed. For endothelial permeability assays, HUVECs were starved in serum-free EBM-2 for $3 \mathrm{~h}$. After starvation, the upper and lower chambers were pretreated with sRAGE for $1 \mathrm{~h}$ and then with Ang II for $4 \mathrm{~h}$. Subsequently, fluorescein isothiocyanate (FITC)-labeled dextran 40 (final concentration of $10 \mathrm{mg} / \mathrm{ml}$ in basal medium) was added as a tracer to the upper chamber, and cells were incubated for $1 \mathrm{~h}$ at $37^{\circ} \mathrm{C}$. After incubation, the medium in the lower chamber was collected into a black 96-well plate, and fluorescence was measured with a fluorescence plate reader (Varioskan Flash; Thermo Fisher Scientific, Waltham, MA, USA) at $485 \mathrm{~nm}$ excitation and $530 \mathrm{~nm}$ emission wavelengths. The permeability of the endothelial monolayer was evaluated by calculating the permeability coefficient of dextran by the following formula: $\mathrm{Pd}=[A] / t \times 1 / A \times V /[L]$, where $[A]$ is the dextran concentration in the bottom chamber, $t$ is the time in seconds, $A$ is the area of the membrane (in $\left.\mathrm{cm}^{2}\right), V$ is the volume of the bottom chamber, and $[L]$ is the dextran concentration in the upper chamber ${ }^{14}$.

\section{Transendothelial electrical resistance}

The transendothelial electrical resistance (TEER) of the HUVEC monolayer was determined using an STX2 
electrode and $\mathrm{EVOM}^{2}$ meter (World Precision Instruments, Sarasota, FL, USA) according to the manufacturer's instructions. HUVECs were seeded in the upper chambers of a 12 -well Transwell insert $(0.4 \mu \mathrm{m}$ pore size $)$ and grown to confluence. The resistance values of multiple Transwell inserts in each experimental group were measured sequentially, and the mean was expressed in common units $\left.(\mathrm{ohm} \mathrm{cm})^{2}\right)$ after the value of a blank, cellfree insert was subtracted. The values were normalized to that of untreated control cells before stimulation.

\section{Immunocytochemistry}

HUVECs were plated in four-well glass chamber slides and cultured to confluence. After sequential treatment with the indicated stimulant, cells were washed in phosphate-buffered saline (PBS) three times, and were then fixed with $4 \%$ paraformaldehyde for $10 \mathrm{~min}$. Cells were washed again with PBS, followed by blocking with $0.5 \%$ bovine serum albumin (BSA) at room temperature and incubation with the appropriate mouse monoclonal VE-cadherin antibody overnight at $4{ }^{\circ} \mathrm{C}$. After three washes with PBS, cells were incubated with FITCconjugated goat anti-mouse antibody at room temperature for $1 \mathrm{~h}$ in the dark. Cells were mounted using DAPIcontaining mounting medium (Santa Cruz Biotechnology). Immunoreactivity signals were visualized by confocal laser scanning microscopy (LSM780; Carl Zeiss, Dresden, Germany).

\section{RNA interference}

A TriFECTa DsiRNA Kit for Diaphanous-related formin-1 (mDia1) was purchased from IDT (Coralville, IA, USA). Scrambled and RAGE siRNAs (siRNA no. 1003394) were purchased from Bioneer (Daejeon, Republic of Korea). Briefly, cells at $60-80 \%$ confluence were transfected with $200 \mathrm{nM}$ RAGE siRNA, scrambled siRNA (negative control), or $20 \mathrm{nM} m$ Dial siRNA using RNAiMax (Invitrogen, Carlsbad, CA, USA) according to the manufacturer's instructions. After $4 \mathrm{~h}$ of transfection, the medium was exchanged with fresh medium.

\section{Western blot analysis}

HUVECs were lysed in RIPA buffer (Biosesang, Gyenoggi-do, Republic of Korea) containing a mixture of protease inhibitors (Thermo Fisher Scientific, Waltham, MA, USA) and centrifuged to extract total protein. Protein concentrations were determined using a BCA protein assay kit (Sigma-Aldrich, St. Louis, MO, USA) according to the manufacturer's instructions, and $25 \mu \mathrm{g}$ of protein was analyzed by $8 \%$ sodium dodecyl sulfatepolyacrylamide gel electrophoresis (SDS-PAGE) (BioRad, Hercules, CA, USA). Membranes with transferred proteins were blocked with 5\% skim milk (BD Biosciences, San Jose, CA, USA) for $1 \mathrm{~h}$ and incubated overnight at
$4{ }^{\circ} \mathrm{C}$ with antibodies against the following proteins: AT1R, RAGE, mDia1, Src, phospho-Src, $\beta$-catenin, phospho$\beta$-catenin, VE-cadherin, phospho-VE-cadherin, HMGB1, NF- $\kappa B$, phospho-NF- $\kappa B, \beta$-actin, and GAPDH. The relative intensities of protein bands were analyzed using ImageJ software (National Institutes of Health, Bethesda, MD, USA).

\section{Reverse transcription polymerase chain reaction}

RNA was isolated from cells using a Ribospin kit (GeneAll, Seoul, Republic of Korea) according to the manufacturer's instructions. Single-stranded complementary DNA (cDNA) was synthesized from $1 \mu \mathrm{g}$ of total RNA with oligo-deoxythymidine (dT) primers using reverse transcriptase (Bio-Rad). The RAGE primer set was purchased from Bioneer (p202745). Additional primer sets were purchased from IDT and validated. The following primer sequences were used: 5'-CAC CAT GTT TTG AGG TTG AGT GAC-3' and 5'-CAG GCT AGG GAG ATT GCA TTT CTG-3' for AT1R; 5'-GGG AGC AAA TCC CAC CTT TA-3' and $5^{\prime}$-GGT CAC AGT ACA ACC CAT AGTC-3' for $m$ Dial; $5^{\prime}$-CGA CCA CTT TGT CAA GCT CA- $3^{\prime}$ and $5^{\prime}$-AGG GGA GAT TCA GTG TGG TG-3' for GAPDH; and 5'-ATT CCG ATA ACG AAC GAG AC-3' and 5'-GCT TAT GAC CCG CAC TTA CT-3' for $18 \mathrm{~S}$ rRNA. The mean values from triplicate $(n=3)$ measurements were used to calculate gene expression after normalization to $18 \mathrm{~S}$ rRNA levels as the internal control.

\section{Measurement of HMGB1}

Culture medium was concentrated from 6 to $1 \mathrm{ml}$ using a centrifugal filter device (Millipore, Billerica, USA). Then, the HMGB1 levels in culture medium were determined by using an ELISA kit (Chondrex, Redmond, WA, USA) according to the manufacturer's instructions and by western blot analysis using $20 \mu \mathrm{l}$ of culture medium.

\section{In vivo study using apolipoprotein $\mathrm{E}$ knockout mice}

Atherosclerosis-prone apolipoprotein $\mathrm{E}$ knockout (ApoE KO) mice, a well-established model of experimental atherosclerosis ${ }^{16,17}$, were used for in vivo studies. Twelve-week-old male ApoE KO mice on a C57BL/6J background were obtained from Jackson Laboratory (Bar Harbor, ME, USA). The animals were housed in a room with a 12-h light/dark cycle and were maintained in temperature-controlled clean racks for 6 weeks. All animal studies and postmortem procedures were approved by the Institutional Animal Care and Use Ethics Committee of Yonsei University (approval reference number: 2014-0350) in accordance with the NIH Guide for the Care and Use of Laboratory Animals.

ApoE KO mice were divided into the following four groups: the untreated control group [saline intraperitoneal 
(IP) injection], Ang II infusion group, Ang II infusion with sRAGE IP injection group, and sRAGE IP injection group. Animals were anaesthetized via IP injection of Zoletil $(30 \mathrm{mg} / \mathrm{kg})$ and Rompun $(10 \mathrm{mg} / \mathrm{kg})$, and Alzet osmotic minipumps (model 2006; DURECT Corporation, Cupertino, CA, USA) were implanted subcutaneously for the infusion of Ang II at a dosage of $1.5 \mu \mathrm{g} / \mathrm{kg} / \mathrm{min}$ for 42 days. sRAGE was injected intraperitoneally daily for 42 days at a dosage of $2 \mu \mathrm{g} /$ mouse/day.

\section{Evans blue administration and quantification}

Animals were perfused with 5\% Evans blue dye in saline through the jugular vein for $45 \mathrm{~min}$. Animals were anesthetized and perfused via the left ventricle with cold PBS, and the aorta was removed. Aortas were measured and quantified using ImageJ software. Evans blue dye was eluted from the aortas by incubation with formamide at $56^{\circ} \mathrm{C}$ for 2 days. The amount of dye was quantified by spectrophotometry at $610 \mathrm{~nm}$.

\section{Transmission electron microscopy}

To examine endothelial permeability, aortic tissues were fixed for $12 \mathrm{~h}$ in $2 \%$ glutaraldehyde-paraformaldehyde in $0.1 \mathrm{M}$ phosphate buffer ( $\mathrm{pH} 7.4$ ), washed in $0.1 \mathrm{M}$ phosphate buffer, and postfixed with $1 \% \mathrm{OsO}_{4}$ dissolved in $0.1 \mathrm{M}$ phosphate buffer for $2 \mathrm{~h}$. Then, samples were dehydrated in ethanol and infiltrated with propylene oxide. Specimens were embedded with a Poly/Bed 812 kit (Polysciences), and 200-250-nm-thick sections were initially cut and stained with toluidine blue (T3260; Sigma-Aldrich, St. Louis, MO, USA) for light microscopy. Ultrathin sections $(70 \mathrm{~nm})$ were cut by a LEICA EM UC-7 (Leica Microsystems, Vienna, Austria) with a diamond knife (Diatome) and were double stained with 6\% uranyl acetate (22400; Electron Microscopy Sciences, Hatfield, PA, USA) for $20 \mathrm{~min}$ and lead citrate (Thermo Scientific, Waltham, MA, USA) for $10 \mathrm{~min}$. All sections were evaluated by transmission electron microscopy (TEM) (JEM1011; JEOL Ltd., Tokyo, Japan) at an acceleration voltage of $80 \mathrm{kV}$.

\section{Statistical analysis}

ImageJ 10.0, SigmaPlot, and GraphPad Prism 5.01 were used for data analysis and visualization. All data are expressed as the means \pm SEMs of at least three independent experiments. Statistical comparisons were performed using Student's $t$-test or one-way analysis of variance (ANOVA) with Tukey's multiple comparisons test. $P$ values of less than 0.05 were considered statistically significant.

\section{Results}

Ang II induced endothelial hyperpermeability in HUVECs

Ang II is known to induce endothelial hyperpermeability. According to our data, as the concentration of Ang
II $(100,300,500$, and $1000 \mathrm{nM}$ for $4 \mathrm{~h})$ increased, the amount of FITC-dextran in the media in the lower chambers also increased, indicating that Ang II dosedependently increased endothelial permeability in HUVECs (Fig. S1a). TEER is another parameter for evaluating cell permeability and is negatively correlated with cell permeability ${ }^{18}$. Ang II $(500 \mathrm{nM})$ also significantly decreased TEER beginning at $4 \mathrm{~h}$ (Fig. S1b). Furthermore, the adherens junction architecture and phosphorylation state of VE-cadherin were examined to evaluate endothelial permeability ${ }^{19,20}$; Ang II caused the disassembly of VE-cadherin junctions (Fig. S2a) and Y731-specific phosphorylation of VE-cadherin (Fig. S2b), indicating disruption of adherens junctions. Taken together, these data indicated that Ang II induced endothelial hyperpermeability in a dose- and time-dependent manner in HUVECs.

\section{RAGE mediates Ang II-induced endothelial hyperpermeability}

To verify the involvement of RAGE signaling in Ang IIinduced endothelial hyperpermeability, siRNA specific for RAGE (Fig. S3) was used to knock down RAGE-mediated signaling. RAGE siRNA significantly suppressed the Ang II-induced upregulation of HMGB1, a major ligand for RAGE known to be upregulated by Ang $\mathrm{II}^{21}$, at both the mRNA (Fig. S4a) and protein (Fig. S4b) levels. During the disassembly of VE-cadherin-mediated cell-cell contacts, kinases such as p21-activated kinase (PAK), Src, and focal adhesion kinase (FAK) facilitate C-tail phosphorylation of VE-cadherin, which can lead to the dissociation of VEcadherin from its accessory molecule $\beta$-catenin ${ }^{22}$.

Since dephosphorylated $\beta$-catenin links cadherin to the actin cytoskeleton and thereby contributes to endothelial cell-cell junction stabilization ${ }^{23}$, phosphorylation of $\beta$-catenin may indicate an increased risk of VE-cadherin disassembly. According to our data, Ang II significantly increased the phosphorylation of Src, $\beta$-catenin, and VEcadherin, but this increase was significantly attenuated by RAGE siRNA (Fig. 1a). Furthermore, RAGE siRNA attenuated the Ang II-induced disassembly of VE-cadherin (Fig. 1b) and decreased the TEER values of confluent HUVEC monolayers (Fig. 1c). These results suggested that RAGE activation modulates Ang II-induced VEcadherin disassembly and subsequent endothelial hyperpermeability.

\section{Activation of AT1R augments the RAGE signaling cascade}

It has been reported that AT1R stimulation by Ang II increases RAGE expression in diabetic atherosclerosis ${ }^{11}$, suggesting possible crosstalk between AT1R-mediated signaling and RAGE-mediated signaling. Additionally, regarding RAGE signaling in endothelial hyperpermeability, mammalian diaphanous 1 (mDia1), a member of 


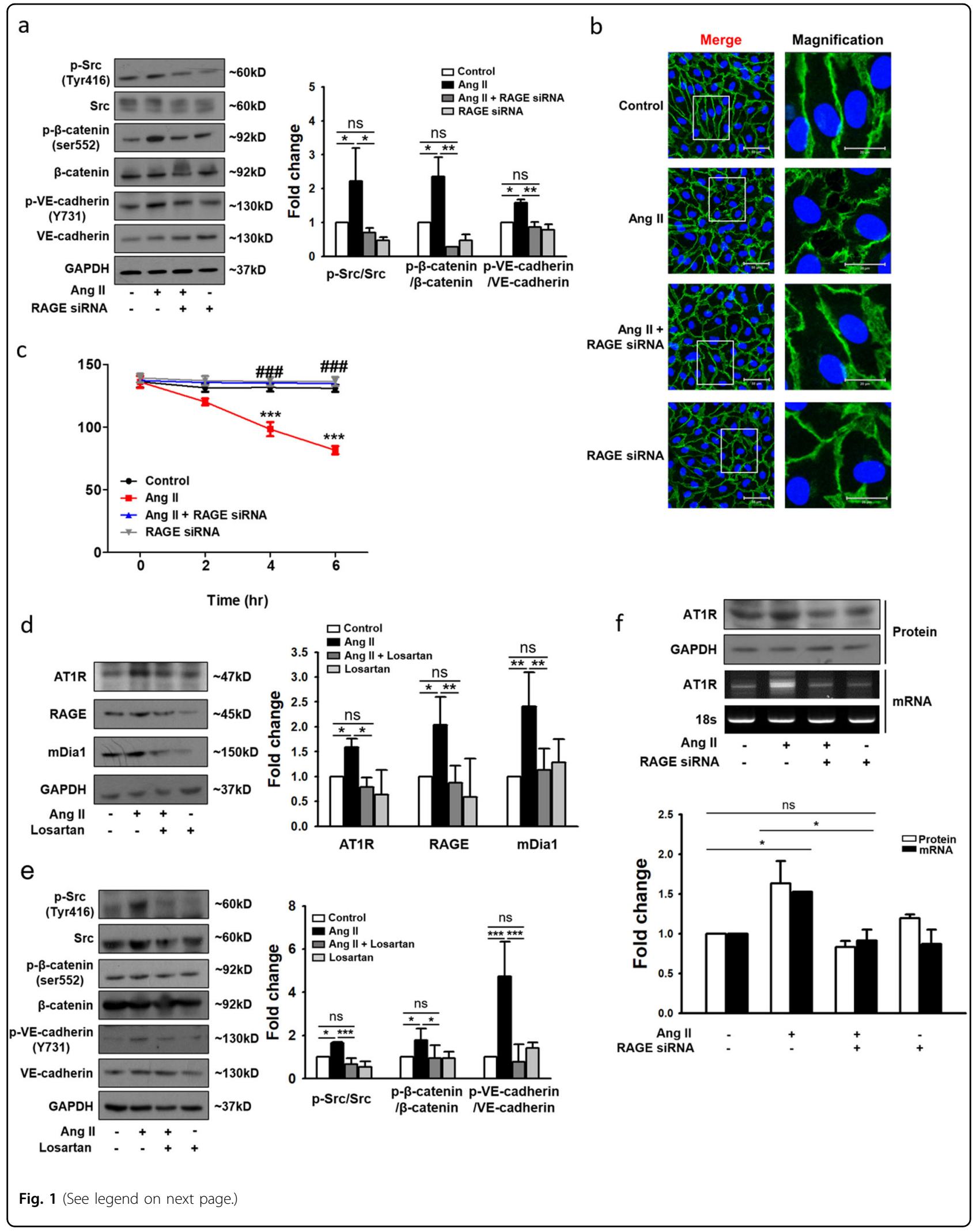


(see figure on previous page)

Fig. 1 Importance of the AT1R-RAGE axis in Ang II-induced endothelial hyperpermeability in HUVECs. a Changes in the phosphorylation of VE-cadherin (Y731), Src (Tyr416), and $\beta$-catenin (Ser552) in HUVECs following transfection with RAGE siRNA and treatment with Ang II. Expression values were normalized to those of VE-cadherin, Src, and $\beta$-catenin ( $n=4$ for each lane). $\mathbf{b}$ Immunocytochemistry of VE-cadherin (green) and DAPI (nuclei, blue), as examined under a confocal microscope (white scale bars: $50 \mu \mathrm{m}$ in merged images and $20 \mu \mathrm{m}$ in magnified images; $\times 400$ magnification). The main images were selected from representative regions. c HUVECs transfected with siRNA targeting RAGE or with scrambled sequences were incubated for $72 \mathrm{~h}$ and were then stimulated with Ang $\|$ for $6 \mathrm{~h}$. TEER was measured every $2 \mathrm{~h} .{ }^{* * *} p<0.001$ vs. control, ${ }^{\# \# \#} p<0.001$ vs. Ang $\|$ ( $n=3$ for each lane). d, e Protein expression in HUVECs treated with Ang $\|$ and losartan $(10 \mu M)$ alone or in combination for $4 \mathrm{~h}$, as determined by western blotting. The relative values of AT1R, RAGE, and mDia1 expression values were normalized to that of GAPDH ( $n=4$ for each lane). The relative values of phospho-Src, phospho- $\beta$-catenin, and phospho-VE-cadherin expression were normalized to those of Src, $\beta$-catenin, and VE-cadherin, respectively ( $n=3$ for each lane). $\mathbf{f}$ Changes in AT1R protein levels in HUVECs following transfection with RAGE siRNA, as determined by western blotting. Expression was normalized to that of GAPDH (top). Changes in AT1R mRNA expression in HUVECs following transfection with RAGE siRNA, as determined by RT-PCR (bottom). Expression was normalized to that of the $18 \mathrm{~S}$ rRNA gene ( $n=4$ for each lane). The values are presented as the means \pm SEMs. ${ }^{*} p<0.05 ;{ }^{* *} p<0.01 ;{ }^{* *} p<0.001$; ns not significant by one-way analysis of variance (ANOVA) followed by Tukey's multiple comparisons test

the diaphanous-related formin family, was recently identified as a new signaling molecule in AGE-induced endothelial hyperpermeability. Mechanistically, mDia1 transduces signaling via binding to the cytoplasmic domain of RAGE (ctRAGE) $)^{24,25}$.

In the present study, Ang II significantly increased the expression of AT1R, RAGE, and mDia1, but this increase was significantly attenuated by losartan, a well-known selective, competitive AT1R antagonist ${ }^{26}$ (Fig. 1d). Furthermore, Ang II-induced phosphorylation of Src, $\beta$-catenin, and VE-cadherin was significantly attenuated by the AT1R antagonist (Fig. 1e). These data suggested that the activation of AT1R reinforces RAGE signaling at least partially by upregulating major mediators of RAGE signaling such as RAGE itself and mDia1. Additionally, the Ang II-induced increase in AT1R expression was significantly suppressed by RAGE siRNA at both the mRNA and protein levels (Fig. 1f), suggesting that RAGE may relay signals between AT1R activation and transcription, establishing a transactivation circuit composed of AT1R-mediated signaling and RAGE-mediated signaling.

\section{HMGB1 links AT1R-mediated signaling and RAGE- mediated signaling}

A well-known RAGE ligand, HMGB1, can be upregulated by Ang II. In addition, our recent study demonstrated that Ang II-induced HMGB1 secretion is critical for the development of cardiac hypertrophy through RAGE activation ${ }^{27}$. Therefore, it was hypothesized that Ang II-induced AT1R activation increases HMGB1 secretion and that HMGB1 subsequently binds to RAGE, initiating RAGE-mediated signaling. In support of this hypothesis, the amount of HMGB1 secreted into the culture medium was significantly increased by Ang II, and this increase was inhibited by the AT1R antagonist losartan (Fig. 2a). Furthermore, HMGB1 neutralizing antibodies significantly suppressed the Ang II-induced expression of AT1R, RAGE, and mDia1 (Fig. 2b) as well as phosphorylation of VE-cadherin at Y731 (Fig. 2c). These data strongly suggested that HMGB1 is the link that connects AT1R activation with the augmentation of RAGE signaling and subsequent disassembly of VEcadherin.

\section{NF-кB facilitates the key modulators of Ang II-induced endothelial hyperpermeability}

The aforementioned data indicated that Ang II augments RAGE signaling by increasing the expression of not only RAGE but also mDia1, a critical accessory molecule in the transduction of RAGE signals. To verify the importance of mDial in Ang II-induced endothelial hyperpermeability, mDia1-specific siRNA (Fig. S5) was utilized. mDia1 siRNA significantly attenuated Ang IIinduced phosphorylation of Src, $\beta$-catenin, and VEcadherin (Fig. 3a), demonstrating the importance of mDia1 in Ang II-induced endothelial hyperpermeability.

To further investigate the regulatory mechanism of mDial, the effect of RAGE knockdown on mDial expression was examined, and the results indicated that RAGE-mediated signaling is required for Ang II-induced expression of mDial (Fig. 3b, c). Although the transcriptional regulation of mDial remains to be clarified, the transcription of both AT1R and RAGE has been reported to be mediated by the transcription factor NF$\mathrm{KB}^{28-31}$. Furthermore, RAGE activation can lead to NF$\mathrm{KB}$-mediated expression of proinflammatory molecules, including RAGE itself ${ }^{32}$. Therefore, the effect of CAPE, a well-known NF-kB inhibitor (Fig. S6) ${ }^{33}$, on the expression of AT1R, RAGE, and mDia1 was examined. Similar to RAGE siRNA, CAPE significantly attenuated Ang IIinduced expression of AT1R, RAGE, and mDia1 (Fig. 3d). Furthermore, CAPE significantly suppressed Ang IIinduced expression of HMGB1 (Fig. S7). Taken together, these data indicated that NF- $\mathrm{kB}$ may be one of the major transcription factors contributing to the transcription of the key molecules involved in Ang II-induced endothelial hyperpermeability. 


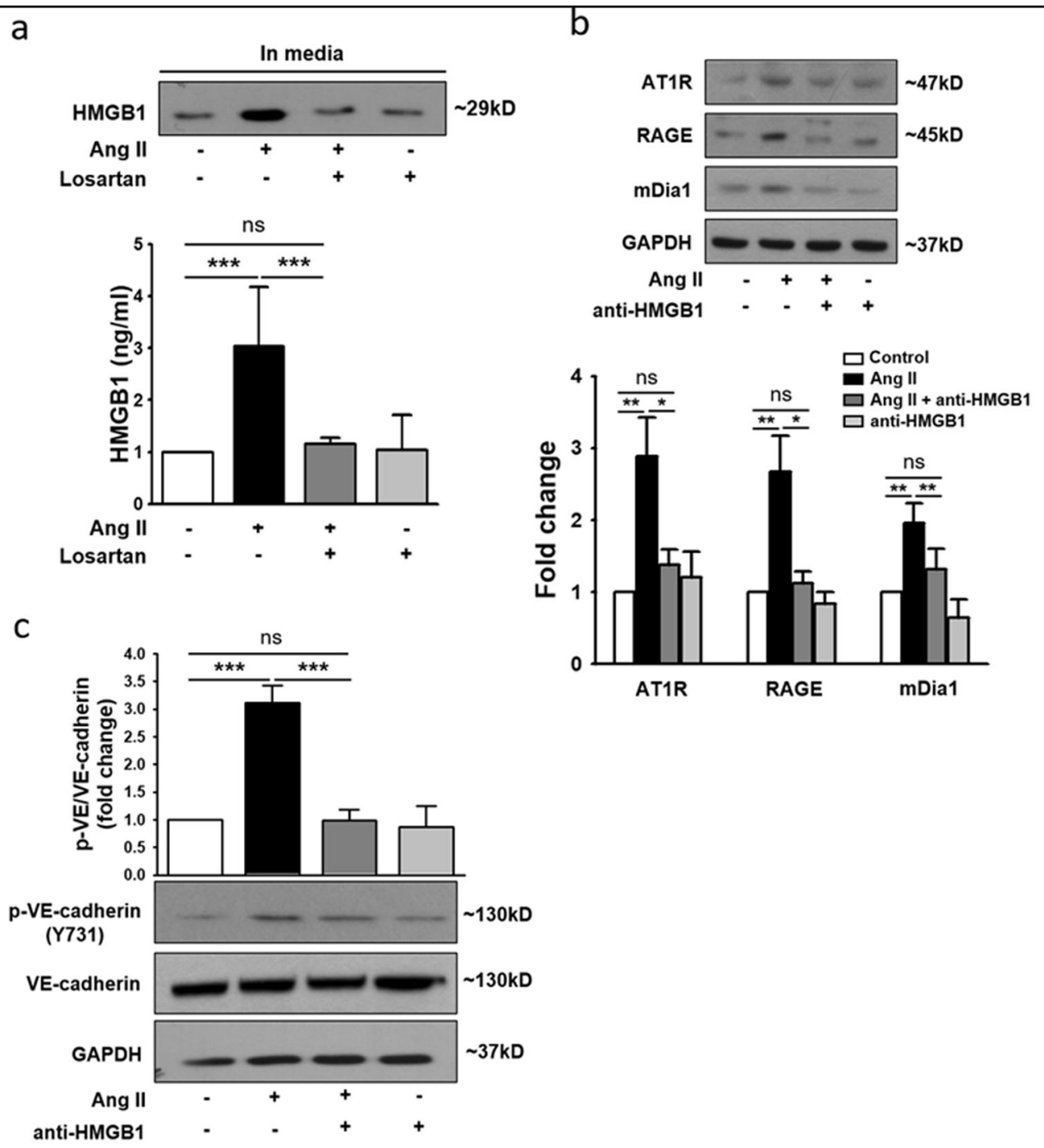

Fig. 2 HMGB1 is an important mediator of AT1R-RAGE signaling. HUVECs were treated with Ang $\|$ in the absence or presence of losartan for $4 \mathrm{~h}$. a HMGB1 release in supernatants was measured by ELISA and western blotting ( $n=4$ for each lane). b, $\mathbf{c}$ HUVECs were incubated with Ang II in the presence or absence of anti-HMGB1-neutralizing antibody $(50 \mathrm{ng} / \mathrm{ml})$ for $4 \mathrm{~h}$ and analyzed by western blotting. The relative values of AT1R, RAGE, and mDia1 expression were normalized to that of GAPDH ( $n=4$ for each lane). The relative values of phospho-VE-cadherin expression were normalized to that of VE-cadherin ( $n=3$ for each lane). The values are presented as the means \pm SEMs. ${ }^{*} p<0.05 ;{ }^{* *} p<0.01$; ${ }^{* * *} p<0.001$; ns not significant by one-way analysis of variance (ANOVA) followed by Tukey's multiple comparisons test

sRAGE attenuates Ang II-induced endothelial hyperpermeability by disrupting the RAGE-mediated signaling cascade

Although RAGE siRNAs significantly attenuated Ang II-induced endothelial hyperpermeability in vitro, the unsolved issues with the use of siRNAs in vivo, such as their low cellular uptake, off-target effects, and instability in serum ${ }^{34}$, could be problematic if a RAGE signaling inhibitor were to be developed as a clinical therapeutic agent. Therefore, as an alternative to RAGE siRNA, sRAGE, which can directly act on circulating RAGE ligands such as HMGB1, was utilized to disrupt RAGE signaling. According to our data, sRAGE significantly attenuated the Ang II-induced decrease in TEER in a dose- and time-dependent manner (Fig. S8), suggesting that it was effective in suppressing the induction of endothelial hyperpermeability by Ang II.

When sRAGE was used alone at a concentration of $2 \mu \mathrm{g} / \mathrm{ml}$, it did not significantly affect the permeability of the endothelial monolayer but significantly attenuated the Ang II-induced increase in the permeability of the endothelial monolayer, as evidenced by the results of the permeability assay using FITC-labeled dextran 40 (Fig. 4a) and the TEER assay (Fig. 4b). The immunocytochemical analysis results showed that sRAGE also prevented Ang II-induced dissociation of VE-cadherin (Fig. 4c) and 


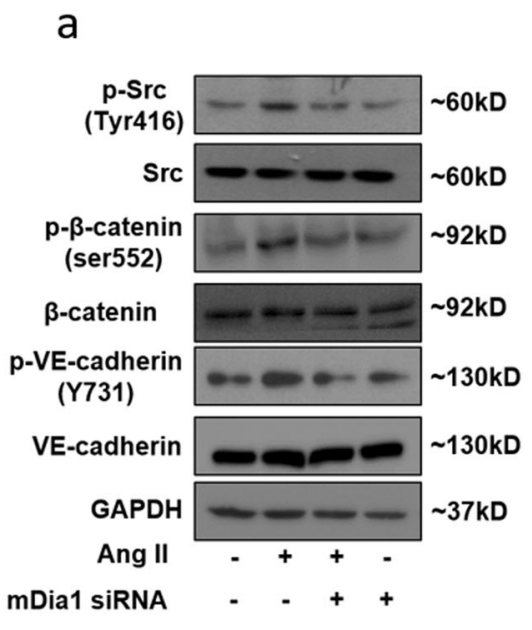

b

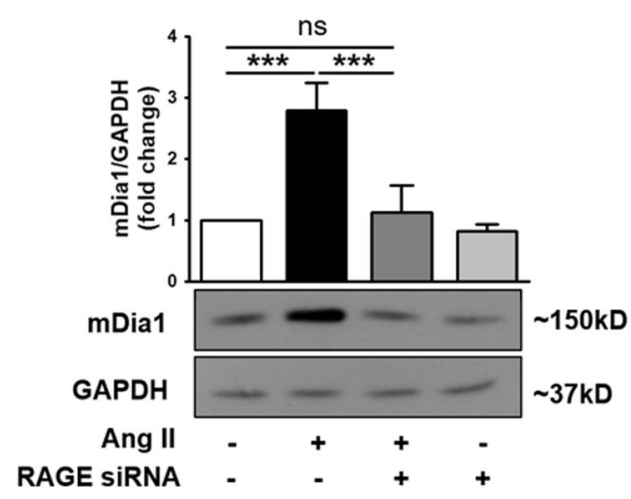

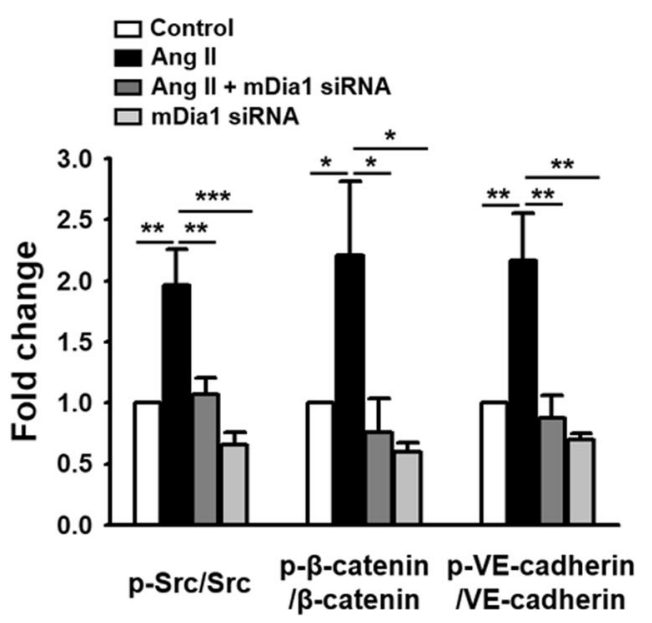

C

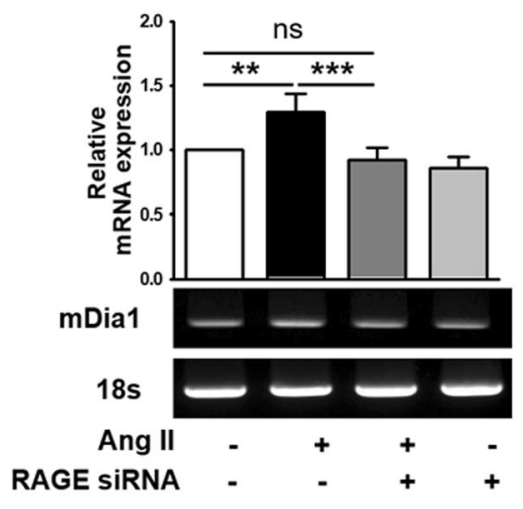

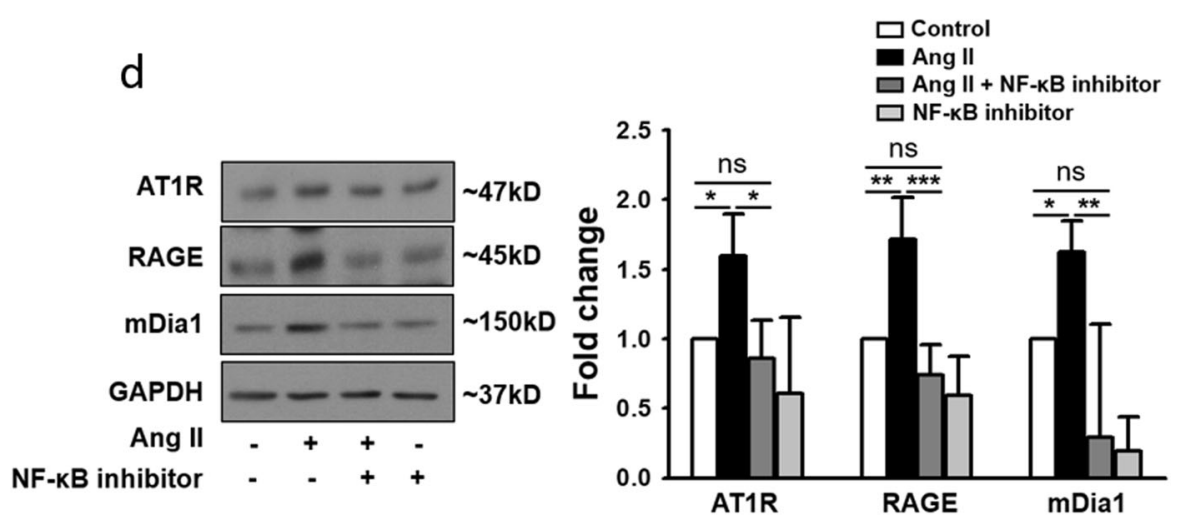

Fig. 3 RAGE regulates Ang II-induced endothelial hyperpermeability via mDia1. a HUVECs were transfected with mDia1 siRNA and cultured in the presence of Ang II for an additional $4 \mathrm{~h}$. Western blot analysis was performed to observe changes in phospho-Src, phospho- $\beta$-catenin, and phospho-VE-cadherin protein expression ( $n=4$ for each lane). b Changes in mDia1 protein levels in HUVECs following transfection with RAGE siRNA, as determined by western blotting. Expression was normalized to that of GAPDH ( $n=3$ for each lane). c Changes in $m D i a 1$ mRNA expression in HUVECs following transfection with RAGE siRNA, as determined by RT-PCR. Expression was normalized to that of the $18 \mathrm{~S}$ rRNA gene ( $n=4$ for each lane). $\mathbf{d}$ HUVECs were treated with Ang $I I$ and the NF-kB inhibitor $(5 \mu \mathrm{g} / \mathrm{ml})$ alone or in combination for $4 \mathrm{~h}$. Protein levels of AT1R, RAGE, and mDia1 in cell lysates were determined by western blotting. Expression was normalized to that of GAPDH ( $n=4$ for each lane). The values are presented as the means \pm SEMs. ${ }^{*} p<0.05 ;{ }^{* *} p<0.01$; ${ }^{* *} p<0.001$; ns not significant by one-way analysis of variance (ANOVA) followed by Tukey's multiple comparisons test 


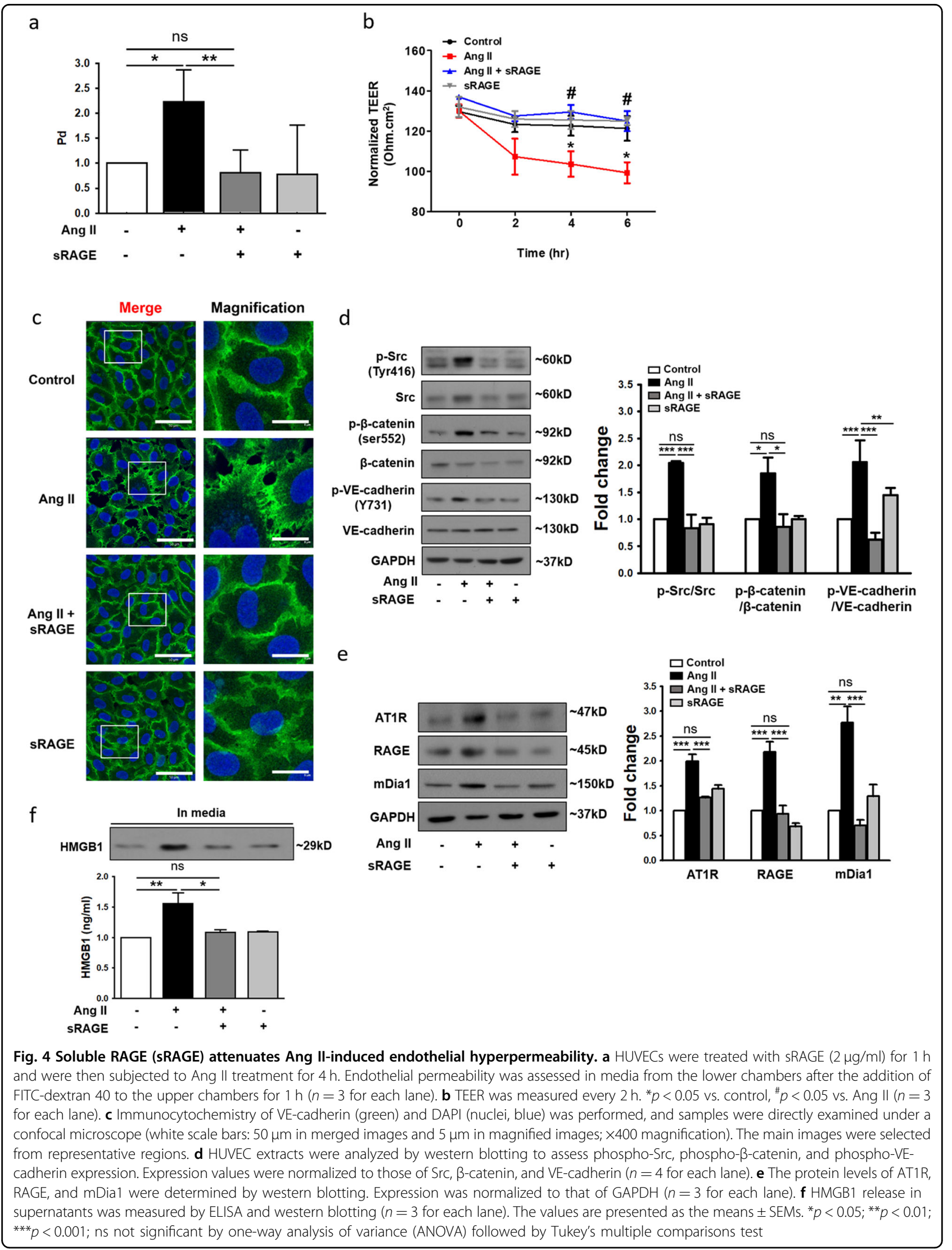


phosphorylation of Src, $\beta$-catenin, and VE-cadherin (Fig. 4d). Furthermore, Ang II-induced upregulation of AT1R, RAGE, and mDia1 was significantly attenuated by sRAGE at both the mRNA level (Fig. S9) and the protein level (Fig. 4e). The amount of HMGB1 secreted following Ang II stimulation was also decreased by sRAGE treatment (Fig. 4f), possibly due to the suppression of Ang IIinduced HMGB1 mRNA synthesis (Fig. S10). These results indicated that the effect of sRAGE was comparable to that of RAGE siRNA in terms of both disrupting the RAGE-mediated signaling cascade and preventing Ang IIinduced endothelial hyperpermeability in vitro.

\section{In vivo delivery of SRAGE attenuates Ang II-induced endothelial hyperpermeability of the aorta in ApoE KO mice}

The Evans blue leakage assay is a well-accepted method for evaluating vascular permeability ${ }^{35}$. As shown in Fig. 5a, vascular permeability was prominently increased by Ang II infusion compared to that in the control group, whereas in vivo delivery of sRAGE dramatically attenuated Ang IIinduced vascular hyperpermeability. This finding was quantitatively confirmed by calculating both the Evans blue-positive area with respect to the total area (Fig. 5b) and the total amount of Evans blue per gram of tissue (Fig. 5c). Closer examination of the endothelium using TEM demonstrated that Ang II infusion caused widening of the intercellular space, which was prevented by in vivo sRAGE delivery (Fig. 5d). Taken together, these results indicated that in vivo delivery of sRAGE effectively suppressed Ang II-induced vascular hyperpermeability in vivo.

\section{Discussion}

Previous studies have demonstrated that both Ang II signaling and RAGE-mediated signaling play important roles in the development of endothelial hyperpermeability ${ }^{15,36}$. Although the importance of crosstalk between signaling through AT1R and RAGE in the pathogenesis of diabetic atherosclerosis has been reported $^{11}$, to our knowledge, such crosstalk between these two signaling pathways in the mediation of endothelial hyperpermeability has not been experimentally confirmed. In the present study, we provided empirical evidence that these two pathways are linked by demonstrating that Ang II increased the production of HMGB1, one of the major ligands for RAGE, in an NF-kB-dependent manner (Fig. $2 \mathrm{a}$, Fig. S7) and that the neutralization of secreted HMGB1 using either HMGB1-specific antibodies (Fig. 2b, c) or sRAGE, a decoy receptor for HMGB1 (Figs. 4 and 5), significantly attenuated Ang II-induced endothelial hyperpermeability. Thus, the present study is the first to report the existence of HMGB1-mediated crosstalk between Ang II signaling and RAGE-mediated signaling in endothelial hyperpermeability.
One of the important physiological functions of the vascular endothelium is to regulate exchange between the blood and interstitial fluid, and meticulous regulation of endothelial permeability is critical for the maintenance of circulatory homeostasis ${ }^{2}$, considering that endothelial dysfunction or endothelial hyperpermeability can exacerbate a variety of diseases, including but not limited to cardiovascular diseases and tumor metastasis ${ }^{37,38}$. To date, several mechanisms of endothelial permeability regulation have been proposed, and Ang II is one of the well-established agents that causes endothelial hyperpermeability ${ }^{39-41}$. Consistent with these previous results, Ang II significantly induced endothelial hyperpermeability, demonstrating the disruption of endothelial cell adherens junctions, a common characteristic of endothelial hyperpermeability (Fig. S2).

VE-cadherin is a component of endothelial adherens junctions that plays a major role in the maintenance of endothelial cell-cell contact by interacting with the cytoskeleton via anchoring molecules such as $\beta$-catenin ${ }^{19,42}$, and its physiological regulation is compromised in Ang II-induced endothelial dysfunction ${ }^{43,44}$. The cytoplasmic tail of VE-cadherin contains at least five tyrosine residues (Y648, Y658, Y685, Y731, and Y733), and phosphorylation of Y731 mediated by the cytoplasmic protein tyrosine kinase $\mathrm{Src}$ is especially important in modulating VE-cadherin activity, since C-tail phosphorylation of VE-cadherin can lead to the dissociation of VEcadherin from its accessory molecule $\beta$-catenin ${ }^{22,45}$. Furthermore, dephosphorylated $\beta$-catenin stabilizes endothelial cell-cell junctions by linking cadherin to the actin cytoskeleton $^{23}$; therefore, phosphorylation of $\beta$-catenin may indicate an increased risk of VE-cadherin disassembly and subsequent endothelial hyperpermeability ${ }^{46,47}$.

In the present study, by demonstrating that knockdown of RAGE via siRNA effectively suppressed Ang II-induced phosphorylation of Src, $\beta$-catenin, and VE-cadherin (Fig. 1a) as well as the disruption of adherens junctions (Fig. 1b) and the decrease in TEER (Fig. 1c), we proved that the RAGEmediated signaling cascade contributes significantly to Ang II-induced endothelial hyperpermeability. It has been reported that RAGE-mediated signal transduction requires interaction of the RAGE cytoplasmic domain with the FH1 domain of the formin mDial (refs. ${ }^{25,48}$ ). Furthermore, according to our data, RAGE not only requires mDia1 to transmit the signal downstream (Fig. 3a) but also positively regulates the expression of $\mathrm{mDia} 1$ in an NF-kB-dependent manner (Fig. 3d), suggesting a positive feedback loop between RAGE and mDia1 to augment the RAGE-mediated signaling cascade. In fact, since the NF- $\mathrm{kB}$ inhibitor also attenuated Ang II-induced expression of AT1R and RAGE (Fig. 3d), it was speculated that NF-kB was a key molecule facilitating an extended positive feedback network involving AT1R, RAGE, and mDial in Ang II-induced endothelial 
a

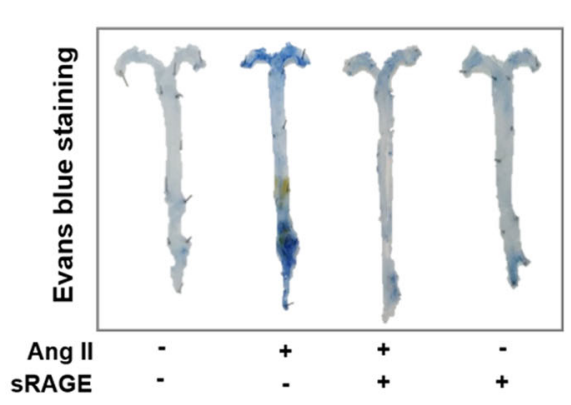

C

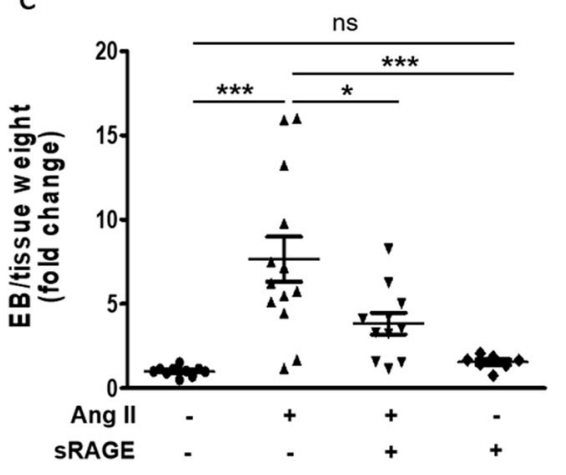

b

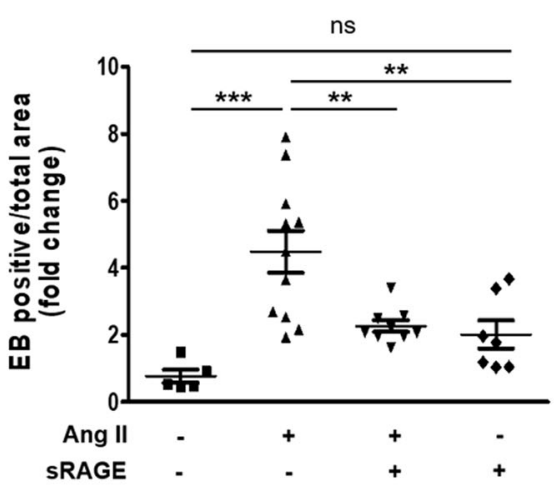

d
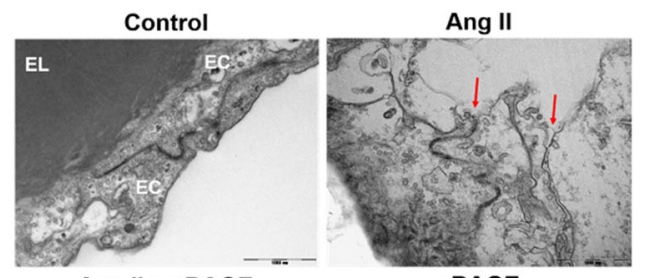

Ang II + sRAGE

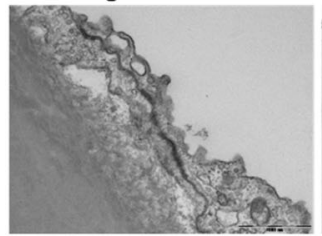

SRAGE

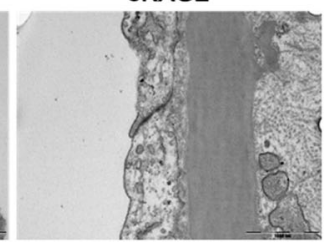

Fig. 5 Effects of sRAGE on Ang II-induced endothelial hyperpermeability in vivo. Twelve-week-old ApoE KO mice were used to investigate endothelial hyperpermeability. Ang II was injected into mice with or without sRAGE for 6 weeks. Then, Evans blue (EB) dye in saline was administered via the jugular vein. a Representative photographs of aortas stained with Evans blue in ApoE KO mice. $\mathbf{b}$ Quantification of positive areas of Evans blue staining in the aortas, as estimated using ImageJ. $\mathbf{c}$ Evans blue dye was eluted from the aortas by incubation with formamide. The amount of dye was quantified by spectrophotometry at $610 \mathrm{~nm}$. $\mathbf{d}$ Representative TEM images of the EC junction area (original magnification, $\times 30,000$ ). EL elastic lamina, EC endothelial cell; scale bar, $1000 \mathrm{~nm}$. Control group, $n=10$; Ang II group, $n=13$; Ang $\|+$ sRAGE group, $n=11$; sRAGE group, $n=7$. The results are representative of at least four separate experiments. The values are presented as the means \pm SEMs. ${ }^{*} p<0.05 ;{ }^{* *} p<0.01$; ${ }^{* * *} p<0.001$; ns not significant by one-way analysis of variance (ANOVA) followed by Tukey's multiple comparison test

hyperpermeability. Previous studies demonstrating RAGEmediated activation of NF-kB ${ }^{27,49}$ and NF-kB-dependent upregulation of $\mathrm{AT}_{1} \mathrm{R}^{28,31}$ and $\mathrm{RAGE}^{29,30}$ also support this speculation. However, without direct empirical evidence, this speculation has to remain a hypothesis yet to be tested, and this insufficiency is one of the limitations of the present study.

Assuming that NF- $\mathrm{B}$ is the key intracellular mediator of Ang II-induced endothelial hyperpermeability, HMGB1 seems to be the critical link that connects AT1Rmediated signaling and RAGE-mediated signaling in the present study. Although HMGB1 is a nonhistone nuclear protein primarily localized in the nucleus ${ }^{50}$, it can be actively released into the extracellular space upon exposure to inflammatory stimuli such as TNF- $\alpha^{51}$. Additionally-and more closely related to the present study-Ang II-induced upregulation of HMGB1 has also been reported ${ }^{27,52}$. These previous observations agree well with our data that clearly demonstrated AT1R-mediated upregulation of HMGB1 (Fig. 2a). Furthermore, yet another previous study reported HMGB1-induced endothelial hyperpermeability ${ }^{53,54}$, and our data demonstrating the effect of the HMGB1-neutralizing antibody on suppressing the upregulation of key molecules (Fig. 2a) and VE-cadherin phosphorylation (Fig. 2c) strongly support the idea that HMGB1 is the critical link in Ang IIinduced endothelial hyperpermeability. However, the observation that both RAGE siRNA (Fig. S4) and the NF-kB inhibitor (Fig. S7) significantly suppressed Ang II-induced upregulation of HMGB1 strongly implies that Ang IIinduced upregulation of HMGB1 was achieved not only by AT1R activation but also by RAGE activation. Multiple steps or multiple mechanisms encompassing both AT1R activation and RAGE activation might have been acting to generate the Ang II-induced upregulation of HMGB1 observed in the present study. Based on the acquired data, the following stepwise signaling events are speculated to occur. 


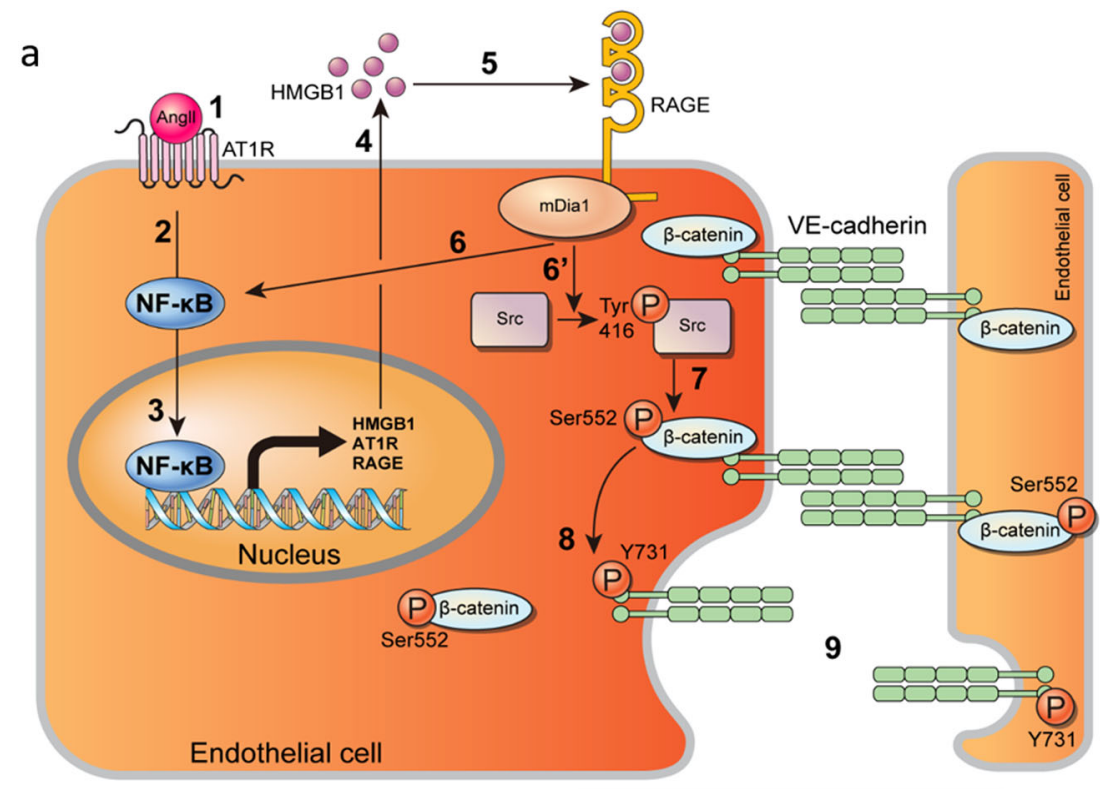

Endothelial permeability 1

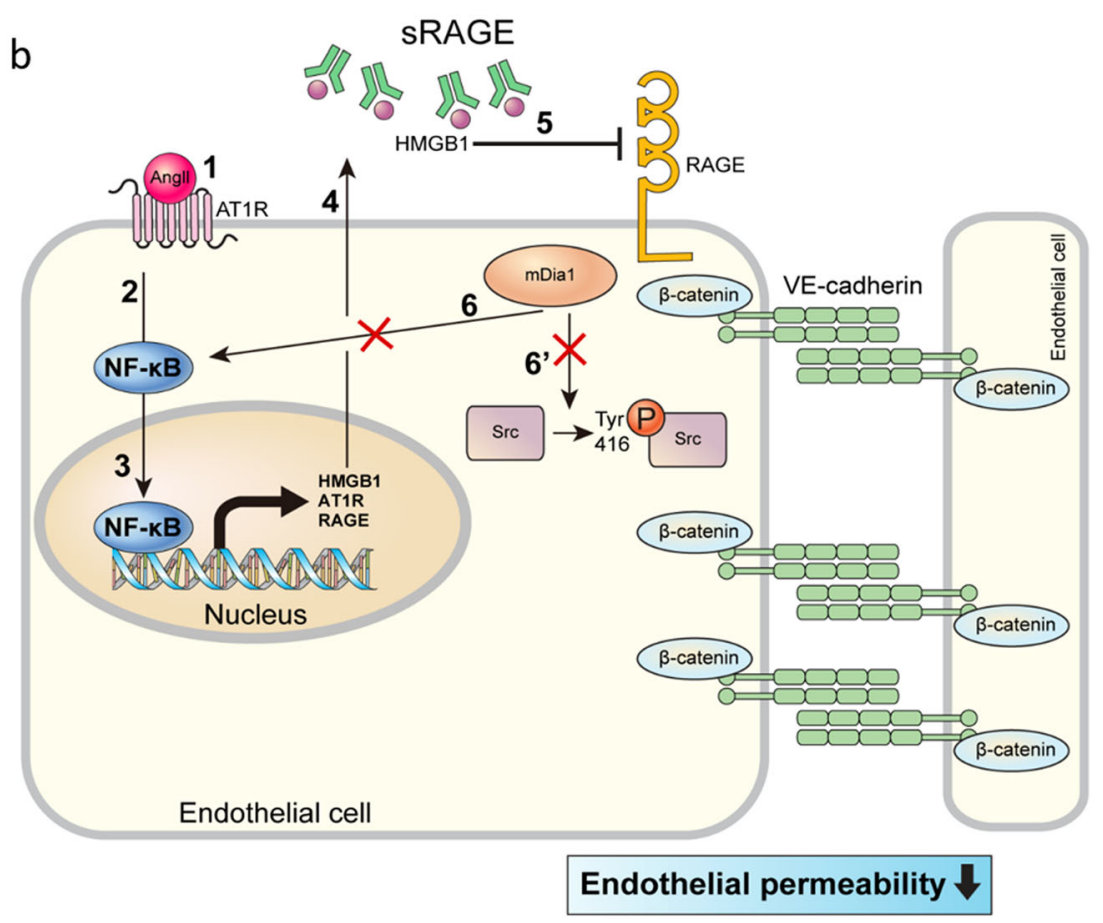

Fig. 6 Schematic diagram of signal transduction pathways involved in Ang II-induced endothelial hyperpermeability via AT1R/RAGE/ mDia1/Src/ $\beta$-catenin/VE-cadherin. a As Ang II binds to and stimulates AT1R, the expression and secretion of HMGB1 can be increased by NF-KB activation. NF-kB-mediated expression of proinflammatory molecules, including RAGE itself, can occur. HMGB1 binds to RAGE, which can induce RAGE-mediated activation of Src/ $\beta$-catenin/NE-cadherin via mDia1. b Blockade of RAGE activation by sRAGE attenuates the Ang II-induced increase in endothelial hyperpermeability by inhibiting RAGE-mediated signaling pathways

First, Ang II activates AT1R, which subsequently activates NF- $\kappa$ B. Activated NF- $\mathrm{kB}$ then increases the expression of HMGB1 along with that of AT1R, RAGE, and mDia1. The increased HMGB1 is then released into the extracellular space to activate RAGE. Finally, activated RAGE subsequently reactivates NF- $\mathrm{B}$ to further increase HMGB1 expression. In this scenario, Ang II can directly increase the expression of HMGB1 by activating 
NF- $\mathrm{KB}$ at the early stage (step 1 in Fig. 6) and indirectly increase the expression of HMGB1 later by activating RAGE (step 4 in Fig. 6). Therefore, blocking RAGE signaling via siRNA or NF- $\mathrm{KB}$ inhibition could at least partially suppress Ang II-induced upregulation of HMGB1. Additionally, Ang II-induced hyperacetylation of HMGB1 might cooperate with the above mechanism to enhance the release of HMGB1 following Ang II stimulation $^{55}$. However, these proposed mechanisms were not examined in detail in the present study, another major limitation of this study. A systematic elucidation of the underlying mechanisms, including the NF- $\kappa B-$ dependent upregulation of key molecules mentioned above, would make a very interesting subject for further studies.

As our data demonstrate, knockdown of the RAGE signaling cascade using RAGE-specific siRNA effectively attenuated Ang II-induced endothelial hyperpermeability in vitro (Fig. 1a-c). However, there is concern about using siRNAs in in vivo studies, although using siRNA in vivo would have been ideal for the present study. In contrast to in vitro experiments in which most variables are controllable, in vivo conditions are difficult to control due to the excessive number of uncontrollable variables. Furthermore, especially for siRNAmediated knockdown in vivo, unsolved issues such as the low cellular uptake, off-target effects, and instability in serum still exist ${ }^{34}$. By using RAGE knockout animals ${ }^{14}$ instead of siRNA-mediated knockdown, these limitations could have been avoided. However, unfortunately, this animal model exceeded the budget; thus, sRAGE was utilized as the second-best option in the present study. Since sRAGE is expected to act on circulating RAGE ligands such as HMGB1, delivering sRAGE via IP injection was thought to be a much more reliable approach than RAGE knockdown using siRNA, which has to first enter endothelial cells to exert the expected effect. Thus, if a RAGE signaling inhibitor were to be developed as a clinical therapeutic agent, delivering peptides would be more effective than delivering siRNA. Additionally, since the main aim of the present study was to suppress RAGE-mediated signaling rather than the expression of RAGE itself, we believe that using sRAGE rather than siRNA sufficiently serves the purpose and is well justified.

sRAGE is a matrix metalloproteinase-cleaved form of RAGE released from the cell surface that functions as an extracellular decoy for RAGE ligands; sRAGE has been implicated in chronic diseases and suggested to be a therapeutically useful biomarker for diseases involving the activation of RAGE signaling cascades ${ }^{56,57}$. The therapeutic potential of sRAGE has been demonstrated in various in vivo disease models, including models of vascular inflammation, diabetes, retinal vascular/neuronal dysfunction, and cardiomyocyte hypertrophy $^{27,58,59}$. However, to our knowledge, its therapeutic potential in Ang II-induced endothelial hyperpermeability has not been empirically examined. Therefore, the present study is the first to investigate the therapeutic potential of sRAGE in the prevention of endothelial hyperpermeability. According to our data, sRAGE effectively attenuated Ang II-induced endothelial permeability in vitro (Fig. 4). Additionally, in the in vivo study using Ang II-infused atherosclerosisprone apolipoprotein $\mathrm{E}$ knockout mice, sRAGE significantly decreased the Evans blue-positive areas compared to those in the Ang II-infused or the salineinfused group, effectively maintaining the integrity of cell-cell contacts (Fig. 5).

The present study provided strong evidence for HMGB1-mediated crosstalk between AT1R-mediated signaling cascades and RAGE-mediated signaling cascades in Ang II-induced endothelial hyperpermeability (Fig. 6). In the clinical context, the results of this study indicate that disrupting the crosstalk between AT1R signaling and RAGE signaling can be an effective means to control Ang II-induced endothelial dysfunction and that sRAGE-mediated blockade of RAGE signaling has significant therapeutic potential. To fully address the unanswered issues, further studies are warranted.

\section{Acknowledgements \\ This work was supported by the Basic Science Research Program through National Research Foundation of Korea (NRF) grants funded by the Korean government (NRF-2015R1A2A2A01007346 to S.P., NRF-2018R1A1A1A05078230 to S.L., and NRF- $2016 R 1$ C1B2016115 to M.S.) and by the Korea Health 21 R\&D Project, Ministry of Health \& Welfare, Republic of Korea (HI08C2149). We thank Mr. Dong-Su Jang, Research Assistant, Department of Anatomy, Yonsei University College of Medicine, Seoul, Korea, for his help with the figures.}

\section{Author details}

${ }^{1}$ Graduate Program in Science for Aging, Yonsei University, Seoul 120-752, Korea. ${ }^{2}$ Integrative Research Center for Cerebrovascular and Cardiovascular Diseases, Yonsei University College of Medicine, Seoul 120-752, Korea. ${ }^{3}$ Department of Pathology, The Catholic University of Korea, Incheon St. Mary's

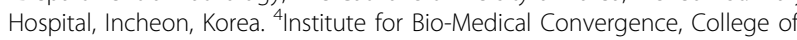
Medicine, Catholic Kwandong University, Gangneung, Gangwon-do 25601, Korea. ${ }^{5}$ Cardiovascular Research Institute, Division of Cardiology, Yonsei University College of Medicine, Seoul 120-752, Korea

\section{Conflict of interest}

The authors declare that they have no conflict of interest.

\section{Publisher's note}

Springer Nature remains neutral with regard to jurisdictional claims in published maps and institutional affiliations.

Supplementary information accompanies this paper at https://doi.org/ 10.1038/s12276-019-0312-5.

Received: 19 December 2018 Revised: 3 July 2019 Accepted: 8 July 2019. Published online: 27 September 2019 


\section{References}

1. Hadi, H. A., Carr, C. S. \& Al Suwaidi, J. Endothelial dysfunction: cardiovascular risk factors, therapy, and outcome. Vasc. Health Risk Manag. 1, 183-198 (2005).

2. Kumar, P. et al. Molecular mechanisms of endothelial hyperpermeability: implications in inflammation. Expert Rev. Mol. Med. 11, e19 (2009).

3. Bogatcheva, N. V., Dudek, S. M., Garcia, J. G. \& Verin, A. D. Mitogen-activated protein kinases in endothelial pathophysiology. J. Investig. Med. 51, 341-352 (2003).

4. Yang, Z., Li, J., Kong, J. \& Wu, S. Impairment of vascular endothelial function following reperfusion therapy in patients with acute myocardial infarction. J. Int. Med. Res. 41, 1074-1078 (2013).

5. Duran, W. N., Beuve, A. V. \& Sanchez, F. A. Nitric oxide, S-nitrosation, and endothelial permeability. IUBMB Life 65, 819-826 (2013).

6. Husain, K., Hernandez, W., Ansari, R. A. \& Ferder, L. Inflammation, oxidative stress and renin angiotensin system in atherosclerosis. World J. Biol. Chem. $\mathbf{6}$, 209-217 (2015).

7. Mehta, P. K. \& Griendling, K. K. Angiotensin II cell signaling: physiological and pathological effects in the cardiovascular system. Am. J. Physiol. Cell Physiol. 292, C82-C97 (2007).

8. Yang, D. et al. Epigallocatechin gallate inhibits angiotensin II-induced endothelial barrier dysfunction via inhibition of the p38 MAPK/HSP27 pathway. Acta Pharm. Sin. 31, 1401-1406 (2010).

9. Hsu, H. H. et al. Mechanisms of angiotensin II signaling on cytoskeleton of podocytes. J. Mol. Med. (Berl.) 86, 1379-1394 (2008).

10. Crowley, S. D. et al. Stimulation of lymphocyte responses by angiotensin ॥ promotes kidney injury in hypertension. Am. J. Physiol. Ren. Physiol. 295 F515-F524 (2008).

11. Ihara, Y. et al. Upregulation of the ligand-RAGE pathway via the angiotensin II type I receptor is essential in the pathogenesis of diabetic atherosclerosis. J. Mol. Cell Cardiol. 43, 455-464 (2007).

12. Cheng, C. L. et al. Advanced glycation end-products activate the reninangiotensin system through the RAGE/PI3-K signaling pathway in podocytes. Clin. Invest. Med. 35, E282 (2012).

13. Wolfson, R. K., Chiang, E. T. \& Garcia, J. G. HMGB1 induces human lung endothelial cell cytoskeletal rearrangement and barrier disruption. Microvasc. Res. 81, 189-197 (2011).

14. Zhang, W. et al. Role of Src in vascular hyperpermeability induced by advanced glycation end products. Sci. Rep. 5, 14090 (2015).

15. Hirose, A., Tanikawa, T., Mori, H., Okada, Y. \& Tanaka, Y. Advanced glycation end products increase endothelial permeability through the RAGE/Rho signaling pathway. FEBS Lett. 584, 61-66 (2010).

16. Jawien, J. The role of an experimental model of atherosclerosis: apoE-knockout mice in developing new drugs against atherogenesis. Curr. Pharm. Biotechnol. 13, 2435-2439 (2012)

17. Meyrelles, S. S., Peotta, V. A., Pereira, T. M. \& Vasquez, E. C. Endothelial dysfunction in the apolipoprotein E-deficient mouse: insights into the influence of diet, gender and aging. Lipids Health Dis. 10, 211 (2011).

18. Dewi, B. E., Takasaki, T. \& Kurane, I. In vitro assessment of human endothelial cell permeability: effects of inflammatory cytokines and dengue virus infection. J. Virol. Methods 121, 171-180 (2004).

19. Vestweber, D. VE-cadherin: the major endothelial adhesion molecule controlling cellular junctions and blood vessel formation. Arterioscler Thromb. Vasc Biol. 28, 223-232 (2008)

20. Sidibe, A. \& Imhof, B. A. VE-cadherin phosphorylation decides: vascular permeability or diapedesis. Nat. Immunol. 15, 215-217 (2014).

21. Chen, J. et al. Inhibition of neointimal hyperplasia in the rat carotid artery injury model by a HMGB1 inhibitor. Atherosclerosis 224, 332-339 (2012).

22. Gavard, J. Endothelial permeability and VE-cadherin: a wacky comradeship. Cell Adh. Migr. 8, 158-164 (2014).

23. Noda, K. et al. Vascular endothelial-cadherin stabilizes at cell-cell junctions by anchoring to circumferential actin bundles through alpha- and beta-catenins in cyclic AMP-Epac-Rap1 signal-activated endothelial cells. Mol. Biol. Cell 21, 584-596 (2010).

24. Senatus, L. M. \& Schmidt, A. M. The AGE-RAGE axis: implications for ageassociated arterial diseases. Front. Genet. 8, 187 (2017).

25. Zhou, X. et al. Mdia1 is crucial for advanced glycation end product-induced endothelial hyperpermeability. Cell Physiol. Biochem. 45, 1717-1730 (2018).

26. Liu, T. J., Shi, Y. Y., Wang, E. B., Zhu, T. \& Zhao, Q. AT1R blocker losartan attenuates intestinal epithelial cell apoptosis in a mouse model of Crohn's disease. Mol. Med. Rep. 13, 1156-1162 (2016).
27. Lim, S. et al. sRAGE attenuates angiotensin I-induced cardiomyocyte hypertrophy by inhibiting RAGE-NFkappaB-NLRP3 activation. Inflamm. Res. 67, 691-701 (2018).

28. Haack, K. K., Mitra, A. K. \& Zucker, I. H. NF-kappaB and CREB are required for angiotensin II type 1 receptor upregulation in neurons. PLOS ONE 8, e78695 (2013).

29. Kang, R. et al. The Receptor for Advanced Glycation End-products (RAGE) protects pancreatic tumor cells against oxidative injury. Antioxid. Redox Signal. 15, 2175-2184 (2011)

30. Li, J. \& Schmidt, A. M. Characterization and functional analysis of the promoter of RAGE, the receptor for advanced glycation end products. J. Biol. Chem. 272, 16498-16506 (1997).

31. Mitra, A. K., Gao, L. \& Zucker, I. H. Angiotensin I-induced upregulation of AT(1) receptor expression: sequential activation of NF-kappaB and Elk-1 in neurons. Am. J. Physiol. Cell Physiol. 299, C561-C569 (2010).

32. Kierdorf, K. \& Fritz, G. RAGE regulation and signaling in inflammation and beyond. J. Leukoc. Biol. 94, 55-68 (2013).

33. Natarajan, K., Singh, S., Burke, T. R., Jr. Grunberger, D. \& Aggarwal, B. B. Caffeic acid phenethyl ester is a potent and specific inhibitor of activation of nuclear transcription factor NF-kappa B. Proc. Natl Acad. Sci. USA 93 9090-9095 (1996).

34. Pecot, C. V., Calin, G. A., Coleman, R. L., Lopez-Berestein, G. \& Sood, A. K. RNA interference in the clinic: challenges and future directions. Nat. Rev. Cancer 11 59-67 (2011).

35. Mammoto, A. et al. Control of lung vascular permeability and endotoxininduced pulmonary oedema by changes in extracellular matrix mechanics. Nat. Commun. 4, 1759 (2013).

36. Bodor, C. et al. Angiotensin II increases the permeability and PV-1 expression of endothelial cells. Am. J. Physiol. Cell Physiol. 302, C267-C276 (2012).

37. Franses, J. W., Drosu, N. C., Gibson, W. J., Chitalia, V. C. \& Edelman, E. R. Dysfunctional endothelial cells directly stimulate cancer inflammation and metastasis. Int. J. Cancer 133, 1334-1344 (2013).

38. Grover-Paez, F. \& Zavalza-Gomez, A. B. Endothelial dysfunction and cardiovascular risk factors. Diabetes Res. Clin. Pract. 84, 1-10 (2009).

39. Newton, C. R., Curran, B. \& Victorino, G. P. Angiotensin II type 1 receptor activation increases microvascular permeability via a calcium dependent process. J. Surg. Res. 123, 33-39 (2005).

40. $\mathrm{Xu}, \mathrm{S}$. Q. et al. Adiponectin protects against angiotensin $\|$ or tumor necrosis factor alpha-induced endothelial cell monolayer hyperpermeability: role of cAMP/PKA signaling. Arterioscler Thromb. Vasc. Biol. 28, 899-905 (2008).

41. Yang, L. X. et al. Role of TRPC1 and NF-kappaB in mediating angiotensin IIinduced $\mathrm{Ca} 2+$ entry and endothelial hyperpermeability. Peptides $\mathbf{3 0}$, 1368-1373 (2009).

42. Giannotta, M., Trani, M. \& Dejana, E. VE-cadherin and endothelial adherens junctions: active guardians of vascular integrity. Dev. Cell 26, 441-454 (2013).

43. Liu, L. et al. Angiotensin II inhibits the protein expression of ZO1 in vascular endothelial cells by downregulating VEcadherin. Mol. Med. Rep. 18, 429-434 (2018).

44. Wu, Z. et al. VE-cadherin involved in the pulmonary microvascular endothelial cell barrier injury induced by angiotensin II through modulating the cellular apoptosis and skeletal rearrangement. Am. J. Transl. Res. 8, 4310-4319 (2016).

45. Oas, R. G. et al. p120-catenin and beta-catenin differentially regulate cadherin adhesive function. Mol. Biol. Cell 24, 704-714 (2013).

46. Hatanaka, K., Simons, M. \& Murakami, M. Phosphorylation of VE-cadherin controls endothelial phenotypes via p120-catenin coupling and Rac1 activation. Am. J. Physiol. Heart Circ. Physiol. 300, H162-H172 (2011).

47. Ali, N. et al. The novel Src Kinase inhibitor M475271 inhibits VEGF-induced vascular endothelial-Cadherin and $\beta$-Catenin phosphorylation but increases their association. J. Pharmacol. Sci. 102, 112-120 (2006).

48. Hudson, B. I. et al. Interaction of the RAGE cytoplasmic domain with diaphanous-1 is required for ligand-stimulated cellular migration through activation of Rac1 and Cdc42. J. Biol. Chem. 283, 34457-34468 (2008).

49. Peng, Y. et al. AGE-RAGE signal generates a specific NF-kappaB RelA "barcode" that directs collagen I expression. Sci. Rep. 6, 18822 (2016).

50. Harris, H. E., Andersson, U. \& Pisetsky, D. S. HMGB1: a multifunctional alarmin driving autoimmune and inflammatory disease. Nat. Rev. Rheumatol. 8, 195-202 (2012).

51. Wang, $\mathrm{H}$. et al. HMG-1 as a late mediator of endotoxin lethality in mice. Science 285, 248-251 (1999). 
52. Nair, A. R., Ebenezer, P. J., Saini, Y. \& Francis, J. Angiotensin II-induced hypertensive renal inflammation is mediated through HMGB1-TLR4 signaling in rat tubulo-epithelial cells. Exp. Cell Res. 335, 238-247 (2015).

53. Zheng, Y. J. et al. Expression of HMGB1 in septic serum induces vascular endothelial hyperpermeability. Mol. Med. Rep. 13, 513-521 (2016).

54. Huang, W. et al. HMGB1 increases permeability of the endothelial cell monolayer via RAGE and Src family tyrosine kinase pathways. Inflammation 35, 350-362 (2012).

55. Zhou, S. et al. Angiotensin II enhances the acetylation and release of HMGB1 in RAW264.7 macrophage. Cell Biol. Int. 42, 1160-1169 (2018).
56. Koyama, H., Yamamoto, H. \& Nishizawa, Y. RAGE and soluble RAGE: potentia therapeutic targets for cardiovascular diseases. Mol. Med. 13, 625-635 (2007).

57. Yan, S. F., Ramasamy, R. \& Schmidt, A. M. Soluble RAGE: therapy and biomarker in unraveling the RAGE axis in chronic disease and aging. Biochem. Pharm. 79 1379-1386 (2010).

58. Wendt, T. et al. RAGE modulates vascular inflammation and atherosclerosis in a murine model of type 2 diabetes. Atherosclerosis 185, 70-77 (2006).

59. Barile, G. R. et al. The RAGE axis in early diabetic retinopathy. Invest. Ophthalmol. Vis. Sci. 46, 2916-2924 (2005). 